\title{
Behmer, Markus \& Katzenberger, Vera (Hrsg.): Vielfalt vor Ort. Privater Rundfunk in Bayern.
}

\section{Bamberg: University of Bamberg Press 2021. 598 Seiten. Preis: $€ 34$}

\author{
Matthias Künzler
}

Angenommen: 22. Dezember 2021 / Online publiziert: 6. Januar 2022

(C) Der/die Autor(en) 2022

Die Einführung des Privatrundfunks in den 1980er-Jahren in Deutschland wird bekanntlich als medienpolitischer „Urknall“ bezeichnet - durchaus zurecht: Mit der damals neuen Institutionalisierungsform veränderten sich Organisationsstrukturen, Produktionsprinzipien, Formate. Neue medienpolitische Fragen traten auf und neue Regulierungsbehörden entstanden.

Angesichts der Tragweite dieser medienpolitischen Entscheidung ist es erstaunlich, dass der kommunikationswissenschaftliche Forschungsstand zum Phänomen „Privatrundfunk" nach wie vor überschaubar geblieben ist. Deshalb ist es sehr begrüßenswert, dass der Sammelband von Markus Behmer und Vera Katzenberger in diese Forschungslücke vorstößt. Der Band hält die Ergebnisse eines von der Bayrischen Landeszentrale für neue Medien (BLM) finanzierten Forschungsprojekts fest. In zahlreichen, vom Herausgeberteam selbst und einer Reihe von weiteren einschlägigen Fachkolleginnen und -kollegen geschriebenen Beiträgen werden relevante Aspekte der historischen Rahmenbedingungen, der vergangenen und gegenwärtigen Struktur des Privatrundfunks, der technischen Entwicklung, Organisationsstrukturen, Arbeits-, Ausbildungsbedingungen und Programmleistungen aufgearbeitet. Ein wichtiger Stellenwert nimmt darin sinngemäß der Bayrische Sonderfall mit seiner Bestimmung ein, dass der Rundfunk im Bundesland in öffentlich-rechtlicher Form verfasst sein muss, was trotzdem eine Vielzahl an unterschiedlichen, privaten Rundfunkunternehmen hervorgebracht hat. Am Ende des Bands findet sich eine Chronik, die einen guten Überblick über die verschiedenen medienpolitisch relevanten Entwicklungen seit den 1970er-Jahren bis heute bietet.

Prof. Dr. Matthias Künzler $(\bowtie)$

Institut für Publizistik- und Kommunikationswissenschaft, Freie Universität Berlin,

Garystr. 55, 14195 Berlin, Deutschland

E-Mail: m.kuenzler@fu-berlin.de 
Die Berücksichtigung dieser Vielfalt an Themenaspekten führt dazu, dass der Band mit knapp 600 Seiten umfassend geraten ist. Dies gereicht ihm jedoch keineswegs zu Nachteil: Die Gliederung ist dank der Zuordnung der Beiträge zu sechs Hauptteilen übersichtlich. Alle Beiträge sind fundiert und gleichzeitig leser:innenfreundlich geschrieben, wozu jeweils auch das zusammenfassende Fazit beiträgt. Obwohl die Studie des Themas wegen sich als Regionalstudie bezeichnen lässt, lohnt sich die Lektüre für alle an Medienentwicklungen und -strukturen interessierte Leserinnen und Leser mit unterschiedlichen Bedürfnissen am Thema. Forschungsinteressierte Personen finden zahlreiche Anregungen für die eigene weitere Forschung, etwa zur Messung der Qualität von lokalen Rundfunkinhalten, zur Frühphase der aus Italien einstrahlenden Piratensender oder zur Entwicklung lokalregionaler Onlineangebote. Studierende finden übersichtliche Einführungen, auch in die medientechnische Entwicklung und deren Folgen für die Ausgestaltung der Rundfunklandschaft. Medienpolitisch aktive Personen dürften an den Untersuchungen zur Ausgestaltung der Medienförderung interessiert sein, die angesichts des eher schrumpfenden Werbevolumens für publizistische Anbieter in den nächsten Jahren im öffentlichen Diskurs an Bedeutung gewinnen wird.

Selbstverständlich gibt es einzelne Aspekte, deren Ausgestaltung ich mir als Leser auch anders hätte vorstellen können. So scheinen mir einzelne Beiträge insbesondere zu Beginn des Sammelbands aus einer „Insiderperspektive“ geschrieben worden zu sein, die davon ausgeht, dass die konkrete Ausgestaltung des Bayrischen Sondermodells bereits bekannt ist. Hier wäre ein einleitender Beitrag, der dieses Modell schildert, mit einer Einschätzung des Herausgeberteams, inwiefern sich dieses Modell in der faktischen Ausgestaltung von den Modellen zur Institutionalisierung der Rundfunkregulierungsbehörden und des Privatrundfunks in den anderen Bundesländern tatsächlich unterscheidet, wünschenswert gewesen. Diese Aspekte kommen zwar in weiteren Beiträgen zur Sprache. Eine integrale Ausgestaltung oder, in der Onlineversion, eine Verlinkung zu den Textstellen in die (Unter-)Kapitel der entsprechenden Beiträge wären ein hilfreicher Service. An wenigen Stellen mag es etwas ungewöhnlich erscheinen, dass einzelne Unterkapitel von eineinhalb Seiten mit einer eigenen Autorin ausgewiesen werden. Ebenfalls ließe sich diskutieren, ob eine noch stärker theoriegeleitete Perspektive den Erkenntnisgewinn und die internationale Anschlussfähigkeit der Studie nicht erhöht hätten. Vermutlich wäre dies jedoch eine Überfrachtung des Vorhabens gewesen, da die Sammlung des Datenmaterials in unveröffentlichten und wenig systematisierten Sammlungen/Archiven und die Generierung von Daten, zum Beispiel durch Interviews mit Beteiligten, bereits ein sehr verdienstvolles Unterfangen und die Grundlage für weitere Forschung zum Themengebiet überhaupt sind.

Deshalb ist zu hoffen, dass die Forschung an solchen Regionalstudien weitergeht. Das Herausgeberteam stellt in seiner Einleitung in Aussicht, dass die 101 durchgeführten Zeitzeugengespräche weiterbearbeitet und der Forschung zur Verfügung gestellt werden. Der Band bietet auch einen Ansporn, ähnliche Studien zum Privatrundfunk in anderen Bundesländern durchzuführen. Aus Sicht der komparativen Forschung wäre dies von großem Interesse, böten solche weiteren Studien doch die Grundlage, Gemeinsamkeiten und Unterschiede im föderalen Medienlabor und dadurch besonders erfolgversprechende Modelle herauszuarbeiten. Dies wäre 
nicht zuletzt die Voraussetzung, noch stärker als bisher auf empirischer Grundlage einen integrativen Blick auf die Rundfunkentwicklung und -struktur zu erhalten und Aussagen zu treffen, inwiefern die mit der Institutionalisierung des Privatrundfunks verbundenen (und sich verändernden) Hoffnungen, Befürchtungen und Erwartungen eingetreten sind.

Funding Open Access funding enabled and organized by Projekt DEAL.

Open Access Dieser Artikel wird unter der Creative Commons Namensnennung 4.0 International Lizenz veröffentlicht, welche die Nutzung, Vervielfältigung, Bearbeitung, Verbreitung und Wiedergabe in jeglichem Medium und Format erlaubt, sofern Sie den/die ursprünglichen Autor(en) und die Quelle ordnungsgemäß nennen, einen Link zur Creative Commons Lizenz beifügen und angeben, ob Änderungen vorgenommen wurden.

Die in diesem Artikel enthaltenen Bilder und sonstiges Drittmaterial unterliegen ebenfalls der genannten Creative Commons Lizenz, sofern sich aus der Abbildungslegende nichts anderes ergibt. Sofern das betreffende Material nicht unter der genannten Creative Commons Lizenz steht und die betreffende Handlung nicht nach gesetzlichen Vorschriften erlaubt ist, ist für die oben aufgeführten Weiterverwendungen des Materials die Einwilligung des jeweiligen Rechteinhabers einzuholen.

Weitere Details zur Lizenz entnehmen Sie bitte der Lizenzinformation auf http://creativecommons.org/ licenses/by/4.0/deed.de.

Prof. Dr. Matthias Künzler ist Professor für Kommunikationspolitik und Medienökonomie an der Freien Universität Berlin. 